$\underset{\text { clinical }}{\text { nephron }}$

Practice
Nephron 2019;143:154-157

DOI: 10.1159/000500093
Received: March 14, 2019

Accepted after revision: April 2, 2019 Published online: April 30, 2019

\title{
Transforming Growth Factor- $\beta$ in the Acute Kidney Injury to Chronic Kidney Disease Transition
}

\author{
Leslie S. Gewin ${ }^{a-c}$ \\ a Division of Nephrology and Hypertension, Department of Medicine, Vanderbilt University Medical Center, \\ Nashville, TN, USA; ${ }^{b}$ Department of Medicine, Veterans Affairs Hospital, Tennessee Valley Healthcare System, \\ Nashville, TN, USA; ' Department of Cell and Developmental Biology, Vanderbilt University, Nashville, TN, USA
}

\section{Keywords}

Acute renal injury · Chronic renal disease · Epithelial cells

\begin{abstract}
Transforming growth factor- $\beta$ (TGF- $\beta$ ) is a key profibrotic growth factor that is activated in acute kidney injury (AKI) and associated with cellular responses that lead to the development of chronic kidney disease (CKD). The persistently injured, de-differentiated tubular epithelial cell is an important mediator of the transition from AKI to CKD. TGF- $\beta$ signaling may perpetuate proximal tubule injury through de-differentiation, cell cycle arrest, and increased susceptibility to apoptosis. In addition, TGF- $\beta$ signaling promotes macrophage chemotaxis, endothelial injury, and myofibroblast differentiation after AKI. Future studies that block TGF- $\beta$ signaling after cessation of AKI are needed to better define its role in the progression of acute to chronic renal injury.
\end{abstract}

(c) 2019 S. Karger AG, Basel

\section{Introduction}

The incidence of acute kidney injury (AKI) and the prevalence of chronic kidney disease (CKD) continue to rise with almost $15 \%$ of Americans having CKD. Several studies have shown that AKI is an independent risk factor for both the development and progression of CKD [1]. The likelihood of AKI transition to CKD depends upon both the severity and persistence of AKI. Although renal function often improves after the initial injury, rodent AKI models demonstrate the development of structural changes that may mediate this AKI to CKD transition. The

Contribution from the AKI and CRRT 2019 Symposium at the 24th International Conference on Advances in Critical Care Nephrology, Manchester Grand Hyatt, San Diego, CA, USA, February 26 - March 1, 2019. This symposium was supported in part by the NIDDK funded University of Alabama at Birmingham-University of California San Diego O'Brien Center for Acute Kidney Injury Research (P30DK079337).

\section{KARGER}

(c) 2019 S. Karger AG, Basel

E-Mail karger@karger.com

www.karger.com/nef
Leslie S. Gewin

Division of Nephrology, Department of Medicine

Vanderbilt University Medical Center, Room S3304 MCN

116121 st Avenue South, Nashville, TN 37232 (USA)

E-Mail l.gewin@vumc.org 


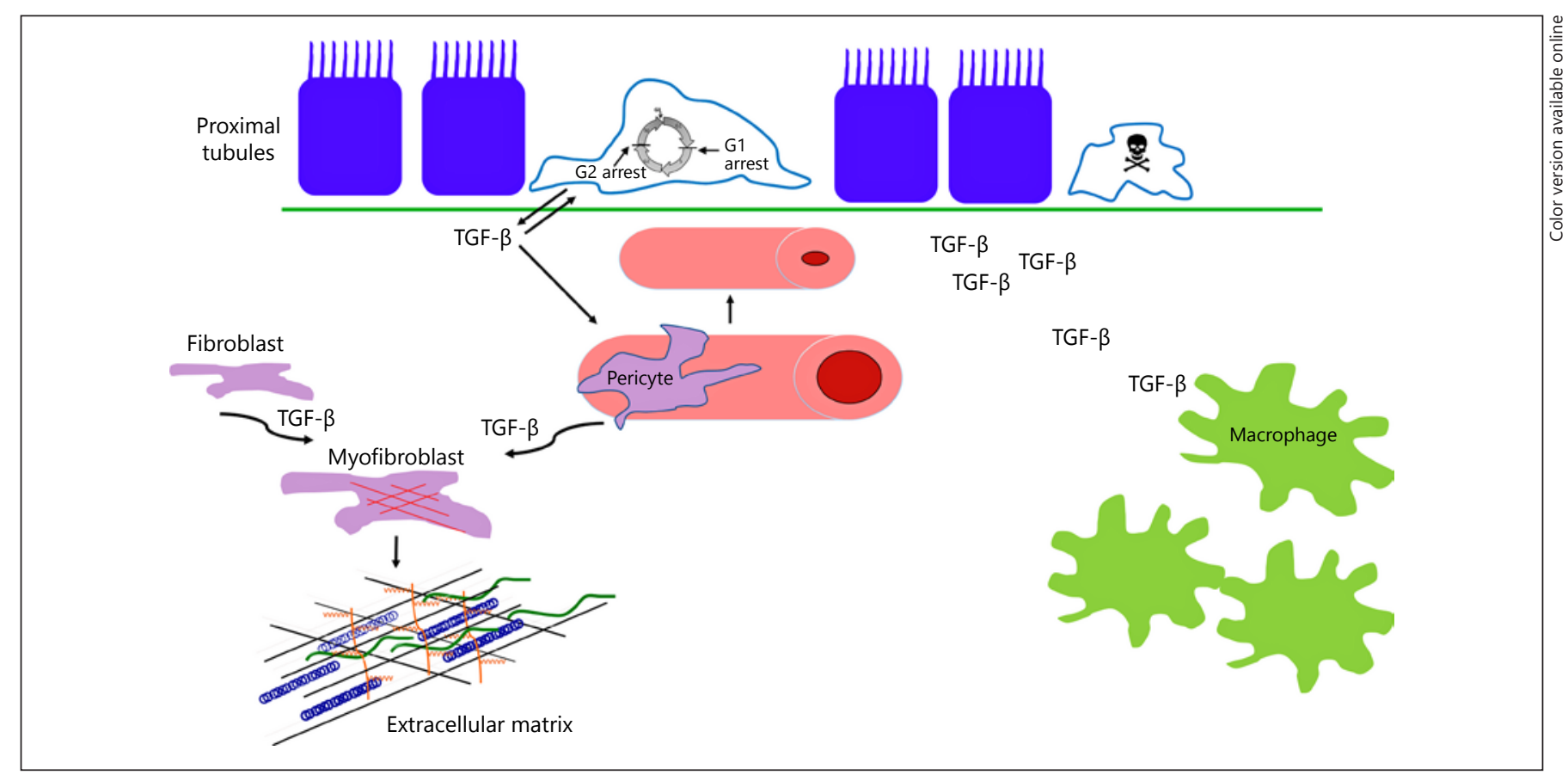

Fig. 1. TGF- $\beta$ signaling mediates effects on different renal compartments that may contribute to the development of CKD after AKI. TGF- $\beta$ is produced by the chronically de-differentiated proximal tubule cell after renal injury. TGF- $\beta$ promotes epithelial dedifferentiation, cell cycle arrest, and sensitizes epithelial cells to apoptosis during injury. TGF- $\beta$ signaling can shorten peritubular capillaries and reduce their patency which may promote CKD progression through worsening hypoxia. TGF- $\beta$ signaling stimulates

mitochondria-dense proximal tubule is the primary cell targeted by AKI. Failure of the proximal tubule to undergo repair and recovery leads to persistently de-differentiated epithelia, which play an important pathophysiologic role in the development of CKD. These chronically injured proximal tubules are potent producers of profibrotic and proinflammatory growth factors, which mediate deleterious responses in surrounding cellular compartments such as the microvasculature and interstitium. Transforming growth factor- $\beta$ (TGF- $\beta$ ) is strongly induced in injured proximal tubule cells, and preclinical studies suggest that autocrine and paracrine TGF- $\beta$ signaling may mediate the progression from AKI to CKD (Fig. 1).

\section{TGF- $\beta$ and the Injured Proximal Tubule}

In AKI, many proximal tubule cells are lost through cell death, and the remaining viable cells undergo de-differentiation and, ideally, proliferate and re-differentiate to re- fibroblasts and pericytes to transform into myofibroblasts, potent producers of extracellular matrix, the hallmark of fibrosis. Finally, TGF- $\beta$ acts as a potent chemoattractant for macrophages and may promote further injury by augmenting macrophage infiltration. One caveat is that most of these TGF- $\beta$-dependent actions were determined by genetic or pharmacologic inhibition of TGF- $\beta$ signaling either before or shortly after acute injury. TGF- $\beta$, transforming growth factor- $\beta$. constitute the tubule epithelia. However, in severe or repeated injury, some de-differentiated epithelial cells become growth arrested, fail to re-differentiate, exhibit altered mitochondrial function and metabolism, and produce large amounts of growth factors like TGF- $\beta$ [2]. The 3 TGF- $\beta$ isoforms $(-1,-2,-3)$ all bind to the type II TGF- $\beta$ receptor, a serine/threonine kinase. Upon ligand binding, the type II receptor heterodimerizes with the Transforming growth factor- $\beta$ type I receptor (T $\beta R I)$, which activates both Smad-dependent and -independent pathways (e.g., MAPK, GTPases) that alter gene expression. TGF- $\beta$ is secreted as an inactive homodimer, but many activators of TGF- $\beta$ (matrix metalloproteases, integrin $\alpha v \beta 6$, thrombospondin-1) are upregulated in renal injury leading to greater TGF- $\beta$ activity with increased injury. TGF- $\beta$ activity can reflect the severity of initial injury, but this cytokine may also play a role in maintaining these injured proximal tubules in a de-differentiated state. TGF- $\beta$ potently induces epithelial de-differentiation by Snail-dependent loss of Ecadherin in vitro [3]. TGF- $\beta$ 's ability to reduce epithelial 
protein expression and increase mesenchymal markers (e.g., $\alpha$-SMA and vimentin) is well documented. Although transient epithelial de-differentiation may be necessary for proliferation and repair, persistent de-differentiation is associated with detrimental responses such as increased matrix and cytokine production. Despite strong in vitro data supporting TGF- $\beta$-dependent de-differentiation, the in vivo data has been somewhat mixed with some studies showing that systemic blockade of TGF- $\beta$ signaling improves tubule differentiation early [4] but others showing few tubular effects after ischemia/reperfusion [5].

TGF- $\beta$ also mediates strongly cytostatic effects on epithelia, which may hinder proliferation that is necessary to repair the injured tubule. The persistence of G2/M-arrested proximal tubules after AKI is associated with a profibrotic epithelial phenotype and the progression to CKD [2]. Although G2/M-arrested epithelial cells secrete large amounts of TGF- $\beta$, it is unclear whether TGF- $\beta$ itself induces arrest at the detrimental G2/M or at G1. Studies have shown TGF- $\beta$-dependent arrest at both checkpoints in vitro with the difference likely related to the dose of TGF- $\beta$ used with lower concentrations associated with G1 arrest (0.5-2 ng/mL) and higher amounts (5-10 ng/ $\mathrm{mL}$ ) with $\mathrm{G} 2 / \mathrm{M}[6]$. How these levels of TGF- $\beta$ in vitro correlate with bioactive TGF- $\beta$ in the injured kidney remains unknown.

Increased proximal tubular cell death is associated with tubular atrophy and development of CKD, and TGF- $\beta$ has been shown by our group and others to sensitize epithelia to apoptosis [7]. Whether this TGF- $\beta$ induced apoptosis worsens the initial response to injury or also contributes to ongoing tubular cell loss after the initial injury needs further investigation. Overexpression of TGF- $\beta 1$ by renal tubular epithelia increased autophagy, severe tubular degeneration and peritubular fibrosis [8]. However, the levels of TGF- $\beta 1$ in this transgenic mouse were likely much higher than those in pathophysiologic states, raising the question of whether TGF- $\beta$ dependent autophagy occurs after AKI and contributes to the CKD transition. Autophagy has protective roles in AKI, but persistent autophagy has been linked with augmented tubular atrophy and interstitial fibrosis [9]. In summary, TGF- $\beta$ signaling in tubular cells promotes epithelial de-differentiation, cell cycle arrest, and reduced survival in vitro and is associated with these responses in vivo. These epithelial effects likely play a role in the AKI to CKD transition, but additional studies that inhibit TGF- $\beta$ signaling after the acute phase of injury are warranted to assess the role of TGF- $\beta$ specifically in AKI to CKD progression.

\section{TGF- $\beta$ and Vascular Endothelia}

Loss of the renal microvasculature is recognized as another important mediator of AKI to CKD progression. Rodent studies show that severe AKI causes microvascular rarefaction and peritubular capillary loss associated with increased TGF- $\beta$ signaling [10]. A systemic T $\beta R I$ antibody administered at the time of I/R and for 5 weeks afterwards showed an attenuated loss in the renal microvasculature without a change in the acute tubular injury [5]. A direct role of TGF- $\beta$ signaling in the vascular endothelium was demonstrated when mice haploinsufficient for T $\beta$ RII had less fibrosis and preserved microvasculature after folic acid administration [11]. These data suggest that TGF- $\beta$ signaling in the vascular endothelia contributes to peritubular capillary rarefaction, potentiating hypoxia and the development of fibrosis.

\section{TGF- $\beta$ and Interstitial Cells}

The tubulointerstitium contains myofibroblasts, the predominant producers of extracellular matrix, and inflammatory cells, and both cell types are important mediators of the AKI to CKD progression. TGF- $\beta$ stimulates fibroblasts and pericytes to develop into myofibroblasts and is a potent inducer of collagen production. This topic has been extensively reviewed previously and is beyond the scope of this mini-review [12]. Many different inflammatory cells contribute to $\mathrm{AKI}$ to $\mathrm{CKD}$ progression, but macrophage infiltration is strongly associated with CKD progression on human biopsies [13]. Macrophages were thought to promote fibrosis through the production of growth factors like TGF- $\beta$, but macrophage-specific deletion of TGF- $\beta 1$ did not attenuate fibrosis after AKI [14]. By contrast, T $\beta$ RII deletion in macrophages did reduce fibrosis after AKI [15]. These conditional knockout mice had less macrophage infiltration with TGF- $\beta$ signaling in macrophages acting as a potent chemoattractant. Thus, increased TGF- $\beta$ production in the injured kidney leads to greater macrophage infiltration, which may promote fibrosis through other factors.

\section{TGF- $\beta$ and AKI to CKD: Unanswered Question}

There is convincing data that TGF- $\beta$ mediates effects on the tubular, endothelial, and interstitial compartments, which can potentially lead to the development of CKD after AKI. However, most of the data come from 
either genetic or pharmacologic approaches in which TGF- $\beta$ activity was inhibited before or shortly after injury. Delaying TGF- $\beta$ inhibition by at least 2 days after injury would better elucidate the role of this important growth factor in the AKI to CKD transition.

\section{Disclosure Statement}

The author has received honoraria and is a consultant for Surrozen, a pharmaceutical company producing a Wnt agonist.

\section{Funding Sources}

Funding for the author was provided by NIDDK R01DK108968-01 and VA Merit 1I01BX003425-01A1.

\section{Author Contributions}

L.S.G. wrote and edited the manuscript.

\section{References}

1 Coca SG, Singanamala S, Parikh CR. Chronic kidney disease after acute kidney injury: a systematic review and meta-analysis. Kidney Int. 2012 Mar;81(5):442-8.

2 Yang L, Besschetnova TY, Brooks CR, Shah JV, Bonventre JV. Epithelial cell cycle arrest in G2/M mediates kidney fibrosis after injury. Nat Med. 2010 May;16(5):535-43.

3 Peinado H, Quintanilla M, Cano A. Transforming growth factor beta-1 induces snail transcription factor in epithelial cell lines: mechanisms for epithelial mesenchymal transitions. J Biol Chem. 2003 Jun;278(23):2111323.

4 Geng H, Lan R, Wang G, Siddiqi AR, Naski $\mathrm{MC}$, Brooks AI, et al. Inhibition of autoregulated TGFbeta signaling simultaneously enhances proliferation and differentiation of kidney epithelium and promotes repair following renal ischemia. Am J Pathol. 2009 Apr; 174(4):1291-308.

5 Spurgeon KR, Donohoe DL, Basile DP. Transforming growth factor-beta in acute renal failure: receptor expression, effects on proliferation, cellularity, and vascularization after recovery from injury. Am J Physiol Renal Physiol. 2005 Mar;288(3):F568-77.
6 Wu DT, Bitzer M, Ju W, Mundel P, Böttinger EP. TGF-beta concentration specifies differential signaling profiles of growth arrest/differentiation and apoptosis in podocytes. J Am Soc Nephrol. 2005 Nov; 16(11):3211-21.

7 Gewin L, Vadivelu S, Neelisetty S, Srichai MB, Paueksakon P, Pozzi A, et al. Deleting the TGF- $\beta$ receptor attenuates acute proximal tubule injury. J Am Soc Nephrol. 2012 Dec; 23(12):2001-11.

8 Koesters R, Kaissling B, Lehir M, Picard N, Theilig F, Gebhardt R, et al. Tubular overexpression of transforming growth factor-beta 1 induces autophagy and fibrosis but not mesenchymal transition of renal epithelial cells. Am J Pathol. 2010 Aug;177(2):632-43.

9 Livingston MJ, Ding HF, Huang S, Hill JA, Yin XM, Dong Z. Persistent activation of autophagy in kidney tubular cells promotes renal interstitial fibrosis during unilateral ureteral obstruction. Autophagy. 2016 Jun;12(6): 976-98.

10 Basile DP, Donohoe D, Roethe K, Osborn JL. Renal ischemic injury results in permanent damage to peritubular capillaries and influences long-term function. Am J Physiol Renal Physiol. 2001 Nov;281(5):F887-99.
11 Xavier S, Vasko R, Matsumoto K, Zullo JA, Chen R, Maizel J, et al. Curtailing endothelial TGF- $\beta$ signaling is sufficient to reduce endothelial-mesenchymal transition and fibrosis in CKD. J Am Soc Nephrol. 2015 Apr;26(4): 817-29.

12 Duffield JS. Cellular and molecular mechanisms in kidney fibrosis. J Clin Invest. 2014 Jun;124(6):2299-306.

13 Eardley KS, Kubal C, Zehnder D, Quinkler M, Lepenies J, Savage CO, et al. The role of capillary density, macrophage infiltration and interstitial scarring in the pathogenesis of human chronic kidney disease. Kidney Int. 2008 Aug;74(4):495-504.

14 Huen SC, Moeckel GW, Cantley LG. Macrophage-specific deletion of transforming growth factor-betal does not prevent renal fibrosis after severe ischemia-reperfusion or obstructive injury. Am J Physiol Renal Physiol. 2013 Aug;305(4):F477-84.

15 Chung S, Overstreet JM, Li Y, Wang Y, Niu A, Wang S, et al. TGF-beta promotes fibrosis after severe acute kidney injury by enhancing renal macrophage infiltration. JCI Insight. 2018 Nov;3(21):pii: 123563. 